\title{
Nanocrystalline diamond particles dispersed by solutions
}

\author{
Kazuyuki Sudoh $^{1}$, Kenji Hirakuri ${ }^{2}$, Kohki Fujioka ${ }^{3,4}$, Noriyoshi Manabe ${ }^{4}$ \\ and Kenji Yamamoto ${ }^{4}$ \\ 1. Graduate School of Advanced Science and Technology, Tokyo Denki University \\ Ishizaka, Hatoyama, Saitama, 350-0394, Japan \\ Fax: 81-49-296-6413, e-mail: kkun81@ff.f.dendai.ac.jp \\ 2. Faculty of Engineering, Tokyo Denki University \\ Fax: 81-3-5280-3648, e-mail: hirakuri@eee.dendai.ac.jp \\ 3. The Jikei University School of Medicine \\ 3-25-8 Nishi-Shinbashi, Minato-ku, Tokyo 105-0003, Japan \\ 4. Research Institute, International Medical Center of Japan \\ 1-21-1 Toyama, Shinjyuku-ku, Tokyo 162-8655, Japan
}

\begin{abstract}
A solution with nanocrystalline diamond (NCD) particles formed using the chemical vapor deposition (CVD) technique and post-treatment process was proposed. After the diamond deposition, the $\mathrm{Si}$ substrate with the deposited diamond particles was immersed in the $\mathrm{HF} / \mathrm{HNO}_{3}$ mixture acid solution to etch the Si substrate. The diamond particles dispersed in the etchant were collected using a membrane filter and re-dispersed onto the ethyl alcohol. Form observations of the diamond particles and grain size distribution before and after the wet etching process were carried out by scanning electron microscope (SEM) and dynamic light scattering (DLS). A mean diamond particle diameter of approximately $250 \mathrm{~nm}$ was observed in the samples. In order to compare the crystalline qualities of the grown diamond, the diamond before and after the wet etching process were analyzed by Raman spectroscopy and electron energy loss spectroscopy (EELS). The Raman spectra with the sharp peak in $1333 \mathrm{~cm}^{-1}$ and the EELS spectra with the peak in $22.9 \mathrm{eV}$ and $34.7 \mathrm{eV}$, oriented by the diamond particles, were confirmed. The results of these analysis indicated that there were no damage to the diamond particles by the wet etching process using the mixture acid etchant. Key words: Nano diamond particle, CVD technique, Si wet etching process, Grain size distribution
\end{abstract}

\section{INTRODUCTION}

Diamond is an attractive material for many industrial fields due to its excellent physical, chemical, electrical and optical properties, such as high hardness, high thermal conductivity, wide band-gap energy, high pair mobility of electron and hole, negative electron affinity, chemical inertness, wide band optical transmittance and good biocompatibility [1]. The diamond cutting, drilling grinding and polishing tools are one of the most famous applications in the mechanical field. Similarly, diamond semiconductor and superconductor have been also researched actively [2], [3]. Diamond has led to the high responsible, high heatproof and low power consumptive. It will have achieved the more high performance semiconductor device, compared with electronic devices based in Si or Ge semiconductors.

Synthetic diamond is very important material due to its low cost, high productivity and high quality, in order to use the diamond as industrial material, because natural diamond is too expensive to use in commercial applications. Technology of synthetic diamond have been enhanced a spread of the application area of diamond. In recent, nanocrystalline diamond (NCD) has been particularly expected for optical, tribological and medical applications because of not only its primary properties but also its small diameter and very low surface roughness [4]-[6]. NCD particles are now mainly produced by the techniques oriented by the phase transition of solid carbon, such as explosive detonation, shock compression and high temperature and high pressure method [6]. However, it is difficult to prepare the NCD particles using above mentioned techniques without contamination of impurities, which are graphite or $\mathrm{Fe}, \mathrm{Co}$ and $\mathrm{Ti}$ used as catalyst materials in diamond deposition[7], [8]. On the other hands, NCD thin film has been deposited from carbon gaseous phase by chemical vapor deposition (CVD) method on the solid substrate[9]. The CVD diamond growth has some advantages such as excellent crystalline qualities (low contaminator), diameter or thickness uniformity, surface modification easily and doping the impurities easily [10]. Developing the new fabrication process of NCD dispersed solution using CVD technique, it is significant for enhancing NCD research and developing the new applications such as medical, semiconductor application.

In this work, we have tried to fabricate the NCD particles dispersed in solvent by two step process. The growth of NCD particles was carried out by hot-filament CVD machine on Si substrate. After the diamond growth, samples were immersed in mixture solution of hydrofluoric (HF) acid and nitric $\left(\mathrm{HNO}_{3}\right)$ acid, to etch the Si substrate and separate from NCD particles. The NCD particles dispersed in the mixture acid solution 
were collected by membrane filter and re-dispersed in the solvent. The morphology and elements profile of NCD particles were observed by a scanning electron microscope (SEM). Raman spectroscopy and electron energy loss spectroscopy (EELS) were also used to identify the crystalline quality of particles before/after the wet etching process.

\section{Experimental Procedures}

- diamond growth

The HFCVD technique was used to grow the diamond particles on $\mathrm{Si}$ (100) substrate [11]. The apparatus incorporated a tungsten wire, and used an electrical power generator and an exhaust system. The dimension of the Si substrate was $10 \times 10 \times 0.4 \mathrm{mmt}$ with ultrasonically surface treatment in $250 \mathrm{~nm}$ diamond powder solution. Table 1 shows the growth conditions. The concentration of $\mathrm{CH}_{4}$ was kept at $0.5 \%$ during diamond growth in a total gas pressure at $5 \mathrm{kPa}$ during diamond deposition. The residence time of the source gases, an essential parameter during CVD diamond deposition, was set at the optimum condition of 0.11 seconds for diamond growth on Si substrate [11]. The filament and substrate temperatures were controlled at $2,370 \mathrm{~K}$ and $1,120 \mathrm{~K}$, respectively. To measure the substrate temperature, a Type $\mathrm{K}$ thermocouple was directly placed in contact with the substrate, passing through a hole in the rear of the substrate holder. Growth time was 15 minutes.

Table 1 Growth condition of diamond

\begin{tabular}{cc}
\hline Process parameters & Values \\
\hline Deposition time & $15 \mathrm{~min}$ \\
Gas flow & $300 \mathrm{sccm}$ \\
$\mathrm{CH}_{4} / \mathrm{H}_{2}$ ratio & $0.5 \%$ \\
Pressure & $5 \mathrm{kPa}$ \\
Residence time & $0.11 \mathrm{sec}$ \\
Substrate temperature & $1120 \mathrm{~K}$ \\
\hline
\end{tabular}

\section{-Wet etching process}

After the diamond deposition, Si substrate with grown diamond particles was immersed in the etchant that have made from mixed $30 \% \mathrm{HF}$ solution $10 \mathrm{ml}$ (Wako, 082-03525, initial concentration $46 \%$, Japan) with $30 \%$ $\mathrm{HNO}_{3}$ solution $10 \mathrm{ml}$ (Wako, 141-01366, initial concentration of $60 \%$, Japan) to etch the $\mathrm{Si}$ substrate chemically and separate diamond particles. Fig. 1 shows the schematic diagram of wet etching process for $\mathrm{Si}$ substrate.

The chemical reactions of wet etching process are as follows;

1. Oxidation of $\mathrm{Si}$ : the $\mathrm{HNO}_{3}$ solution plays a role of the oxidant reaction at the mostly outside surface region of Si substrate.

$$
\mathrm{Si}+4 \mathrm{HNO}_{3}=\mathrm{SiO}_{2}+4 \mathrm{NO}_{2}+2 \mathrm{H}_{2} \mathrm{O} \cdots(1)
$$

2. Etching of $\mathrm{SiO}_{2}$ : the HF solution causes the etching reaction of the oxidized region. This reaction occur replacement process between $\mathrm{Si}$ atoms and oxygen atoms.

$$
\mathrm{SiO}_{2}+6 \mathrm{HF}=\mathrm{H}_{2} \mathrm{SiF}_{6}+2 \mathrm{H}_{2} \mathrm{O} \cdots(2)
$$

The NCD particles were separated from Si substrate and dispersed in the etchant. The particles were collected by membrane filter (Millipore, VVLP0905, 100nm VVPP, Ireland Rev.), and re-dispersed in the ethyl alcohol.

(1)

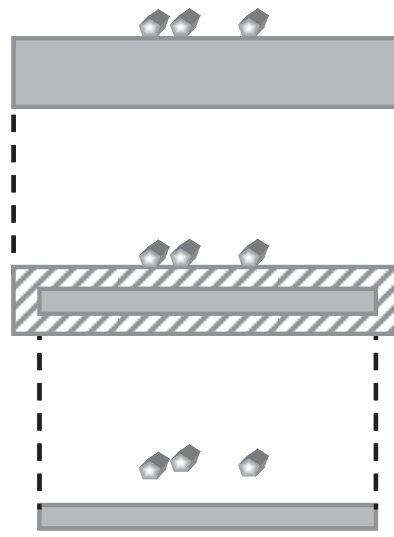

Fig. 1 Schematic diagram of wet etching process.

\section{-Analysis of diamond particles}

The form observation of diamond particles and grain size distribution before/after the wet etching process was observed by SEM (Jeol, Model JSM - 5310LVB, Japan) and dynamic light scattering (DLS) (Malvern, Model Zerasizer Nano ZS, UK). In order to compare the crystalline qualities of the grown diamond, the diamond before/after the wet etching process were analyzed by Raman spectroscopy (Jasco, Model NRS - 2100, Japan) using an argon laser (514.52 nm) and electron energy loss spectroscopy (EELS) using auger electron spectroscope, in acceleration voltage at $2 \mathrm{kV}$ (Jeol, Model JAMP - 7810, Japan). In the diamond analysis of after the wet etching process, the diamond particles were buried in the indium substrate to protect the sample and analyze surely.

\section{Results and discussion}

- diamond form observation and size distribution

Fig. 2 shows the diamond particle before/after the etching process. Diamond particles deposited on the $\mathrm{Si}$ substrate were confirmed in Fig. 2(a), and buried in the Indium substrate was confirmed in Fig. 2(b). Diamond particles that have the mean diameter of approximately $250 \mathrm{~nm}$ were observed. The crystal faces were confirmed at each photograph. It is a feature of excellent crystalline quality diamond.

Fig. 3 shows the grain size distribute observation by DLS. The grain size was distributed from $60 \mathrm{~nm}$ to 1000 $\mathrm{nm}$, and the distribution with the mean diameter of 250 
$\mathrm{nm}$ was obtained. These results showed that the SEM observation and DLS analysis were corresponding. Moreover, the uniformity of the grain size was suggested because the high peak intensity was observed near the mean diameter of diamond particles and it has very shape peak.
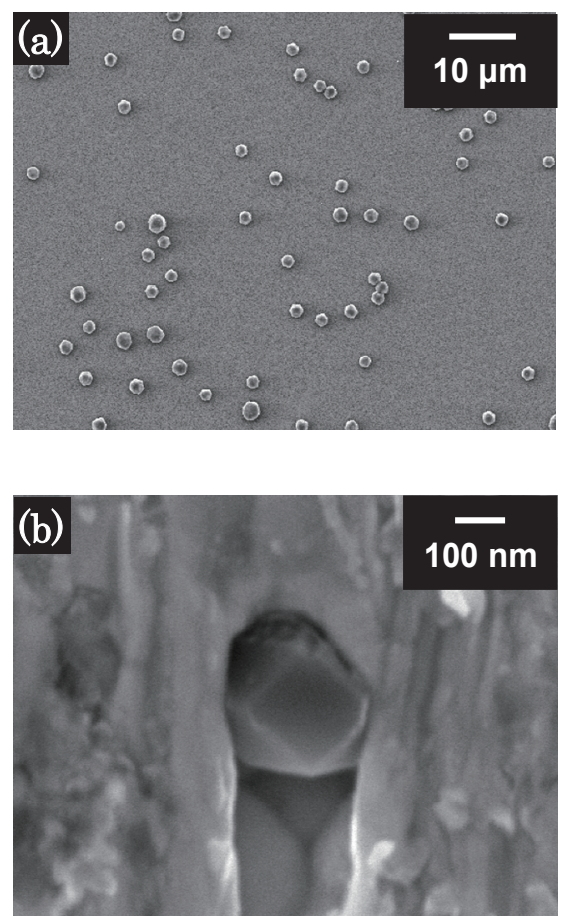

Fig. 2 SEM images of diamond particles (a) on the $\mathrm{Si}$ substrate and (b) in the Indium substrate.

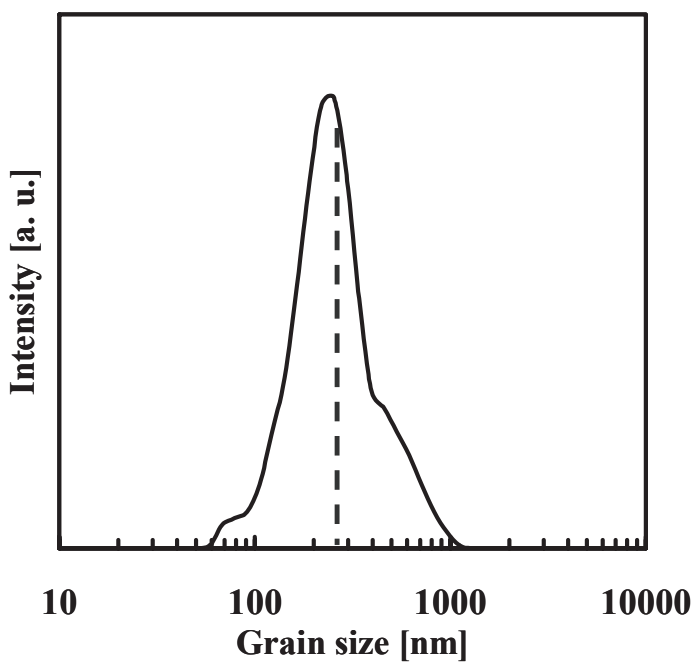

Fig. 3 The grain size distribution of diamond particles.

- Crystalline quality of diamond particles

The Raman spectra of diamond particles before/after the wet etching process are shown in Fig. 4. Each sample had a spectrum that was typical line shape originated by diamond, exhibiting at $1,333 \mathrm{~cm}^{-1}$ as diamond. The FWHM value, which means the crystalline quality, was calculated using the diamond peak occurring in the Raman spectra, and it was shown $14.6 \mathrm{~cm}^{-1}$ at as deposited diamond, and $15.6 \mathrm{~cm}^{-1}$ at the diamond after the etching process. As a result of Raman data analysis, there was no difference of the crystalline quality between the diamond particles on the $\mathrm{Si}$ substrates and that after the wet etching process.

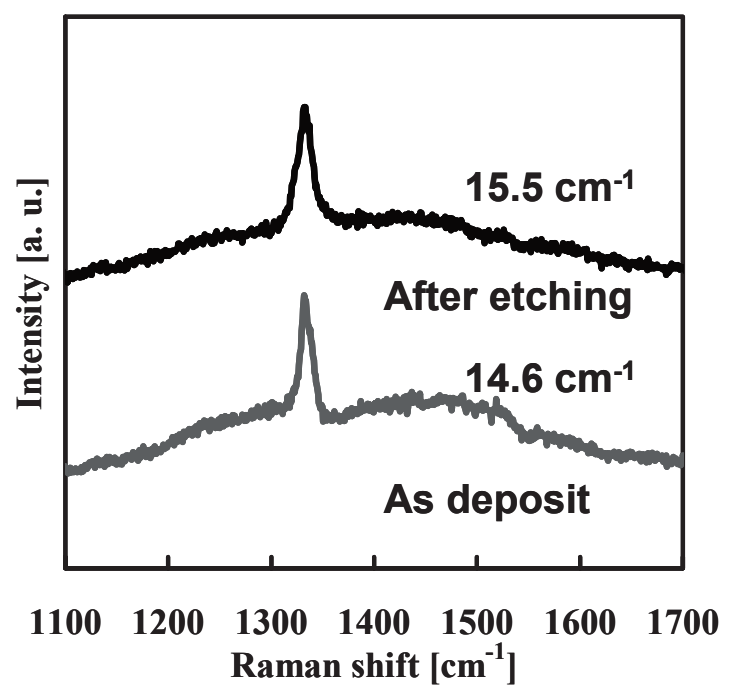

Fig.4 Raman spectra of diamond particles

Fig. 5 shows the EELS spectra of diamond particles before/after the wet etching process. In the EELS analysis for diamond, two typical peaks will be observed. One is the $22.9 \mathrm{eV}$ that oriented by surface plasmon, another is $34.7 \mathrm{eV}$ that oriented by balk plasmon. Similarly, there is a possibility that shows the peak in $2.6 \mathrm{eV}$ that means the graphite. The EELS spectra that have broad $22.9 \mathrm{eV}$ and $34.7 \mathrm{eV}$ peak, and no peak evidence at $2.6 \mathrm{eV}$ were observed in each samples. It suggests that the diamond particles obtained in this study have an excellent crystalline quality.

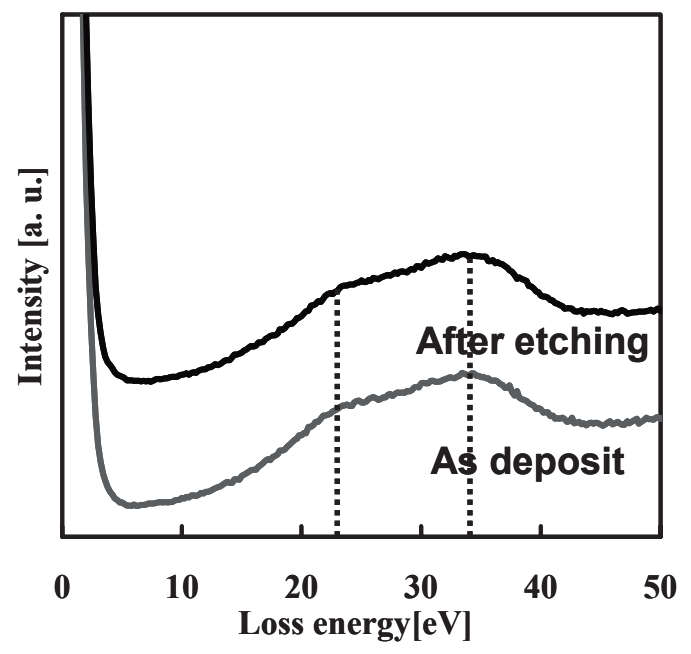

Fig. 5 EELS spectra of diamond particles. 
As the results of Raman spectroscopy and EELS analysis, the diamond particles with good crystalline quality and without containing impurities were obtained. Moreover, it was no difference for diamond quality at before/after the wet etching process. It showed that there was no damage to diamond particles by the wet etching process using mixture acid etchant. And it suggested that the high quality NCD particles without impurities were obtained via this process.

\section{Conclusion}

In this study, we proposed and tried the new fabrication process for NCD particles using CVD technique and mixture acid etchant. As the results, diamond particles with high crystalline quality were successfully obtained in solution. The CVD techniques are very suitable for high crystalline quality diamond deposition, and mixture acid etchant did not cause the corrosion for diamond particles. Using this process, it is fascinating to apply the NCD particles dispersed by solutions for electric, optical and medical applications.

\section{Acknowledgement}

The authors express their gratitude to Mr. Tuneo Toya for valuable technical assistance in this experiment. This study was supported by the Center for Research, Tokyo Denki University.

\section{Reference}

[1] S. E. Coe et al, Diamond Relat. Mater. 9, $1726(2000)$.

[2] S. Almaviva et al, Diamond Relat. Mater. 18, 101 (2009).

[3] S. Wang et al, Diamond Relat. Mater., in press.

[4] K.A. Ten et al, Nucl. Instrum. Method A, in Press.

[5] H. Sumiya, et al, Diamond Relat. Mater. 13, 1771 (2004).

[6] C. Mangeney et al, Diamond Relat. Mater. 17, 1881 (2008).

[7] Chao Wen et al, Mater. Letters 60, 3507 (2006)

[8] A. E. Aleksensky et al, Diamond Relat. Mater. 13, 2076 (2004)

[9] S.J. Askari et al, Vacuum 81, 713 (2007).

[10] I. Sakaguchi et al, Phys. Rev. B 60, 2139 (1999).

[11] T. Kobayashi et al, Diamond Relat. Mater. 8, 2026 (2000).

(Received December 9, 2008; Accepted February 3, 2009) 